\title{
Kinetically Self-Limiting Growth of Ge Islands on Si(001)
}

\author{
Martin Kästner and Bert Voigtländer \\ Institut für Grenzflächenforschung und Vakuumphysik, Forschungszentrum Jülich, 52425 Jülich, Germany
} (Received 31 August 1998)

\begin{abstract}
The evolution of the size and shape of individual $\{105\}$ faceted $\mathrm{Ge}$ islands on $\mathrm{Si}(001)$ is measured with a high temperature scanning tunneling microscope during growth. A slower growth rate is observed when an island grows to larger sizes. This behavior can be explained by kinetically self-limiting growth. A kinetic growth model involving a nucleation barrier for each repeated growth of a new atomic layer on the $\{105\}$ facets agrees with the experimental results for the evolution of the island volume. The experimentally observed shape transition from nearly square shaped islands to elongated islands is described by the kinetic growth model. [S0031-9007(99)08803-1]
\end{abstract}

PACS numbers: 68.55.Jk, 07.79.Cz, 68.65.+g

The self-organized growth of strained islands in heteroepitaxial growth is currently employed to build dense arrays of nanoscale quantum dots [1,2]. Optoelectronic applications are expected due to carrier confinement in the islands and subsequent recombination and emission of photons. In particular, the SiGe system is investigated intensively because of the compatibility with the existing $\mathrm{Si}$ technology. However, it is not yet possible to grow islands of desired size, shape, and density. In order to control the island morphology better, it is important to identify whether the island morphology resembles an energetically stable configuration or whether the island morphology is determined by growth kinetics.

For applications, a narrow island size distribution is very desirable. Recently, a model for the energy of a strained island has been developed which includes elastic strain relaxation, surface energies, and elastic interaction of the edges [3]. Depending on the material parameters, there may be a minimum of the island energy as a function of the volume. Such energetically stable island sizes would favor a narrow size distribution $[1,4]$. Alternatively kinetically self-limited growth may lead to a narrow island size distribution. Self-limitation occurs when larger islands grow slower than smaller ones. Since smaller islands catch up in size a narrow island size distribution will be favored. A kinetic energy barrier for growth on the island facets can lead to self-limiting growth $[5,6]$.

Here we examine the growth of Ge islands with $\{105\}$ facets, the so-called "hut" clusters [7-10]. Since such Ge islands are the smallest observed on $\mathrm{Si}(001)[1,11]$, they are best suited as nanometer sized islands. The size evolution of single $\{105\}$ faceted islands is measured directly during growth using a scanning tunneling microscope (STM). We find direct evidence for self-limiting behavior, i.e., the growth rate is observed to decrease when the Ge islands grow larger. A growth model, including a kinetic energy barrier for the successive overgrowth of the $\{105\}$ facets $[5,6]$, can describe the observed self-limiting growth of the Ge islands. Additionally, this kinetic growth model can explain the experimentally observed shape transition from initially nearly square shaped islands to elongated shapes.

The experiments were carried out with a hightemperature STM, in which the growing surface is imaged continuously during growth. This enables us to study directly the evolution of the size and shape of individual islands as a function of time. We used a beetle-type STM, which is described in detail in Ref. [12]. Evaporation is done continuously while the STM scans the growing film. At a substrate temperature of $575 \mathrm{~K}, \mathrm{Ge}$ was evaporated at a constant growth rate of $0.06 \mathrm{ML} / \mathrm{min}(1 \mathrm{ML}=$ $6.78 \times 10^{14}$ atoms $/ \mathrm{cm}^{2}$ ). After the completion of the wetting layer, three-dimensional Ge islands with $\{105\}$ facets nucleate. The island edges are oriented along $\langle 100\rangle$ directions, i.e., at $45^{\circ}$ to the direction of the reconstruction dimer rows. We observe only completed $\{105\}$ facets. The strain of these islands is partially relaxed elastically, but they are coherent with the substrate lattice (i.e., free of dislocations between the substrate and the hut clusters) [7]. The evolution of these hut clusters is observed by STM during growth. A sequence of STM images is shown in Fig. 1. From the images the volume of the hut clusters can be calculated: $V=\frac{4}{15} s^{3}+\frac{1}{5} s^{2} d$. Figure 2 shows the evolution of the volume of several islands (labeled in Fig. 1) as a function of the total deposited coverage. The initially higher growth rate of individual islands just after the nucleation, indicated by initially large slopes in Fig. 2, decreases when the islands grow to a larger size. In the following, we present a model which shows that kinetically self-limiting growth explains the observed slower growth for larger island sizes [13].

We use a kinetic model for the growth of hut clusters which relies on a barrier for the nucleation of each successive atomic layer on the $\{105\}$ facets [5]. The energy to form a hut cluster can be written as composed of step-step interaction energies. The total energy (per unit length parallel to the steps) to form steps at positions $x_{i}$ can be approximated as [14] 

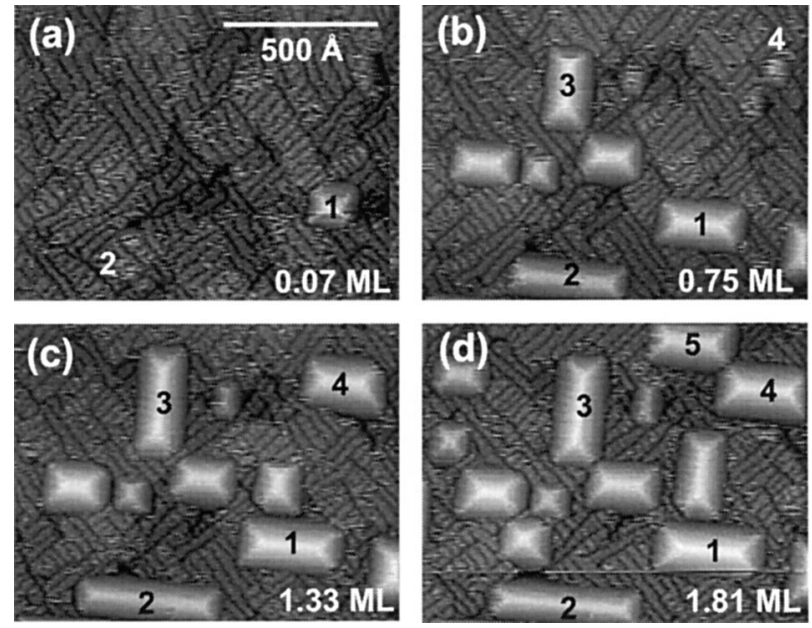

FIG. 1. STM sample images of the hut cluster growth as a function of coverage (beyond the wetting layer). The hut clusters are imaged as rectangular bright areas (image area: $1300 \times 1000 \AA^{2}, T=575 \mathrm{~K}$ ). Identical islands are numbered in (a)-(d). The complete growth sequence is available as a movie [17].

$$
\begin{aligned}
E= & \sum_{i} C_{0}+\sum_{i \neq j} C_{m} \sigma_{i} \sigma_{j} \ln \left(\frac{x_{i}-x_{j}}{a}\right) \\
& +\sum_{i \neq j} C_{d}\left(\frac{x_{i}-x_{j}}{a}\right)^{-2} .
\end{aligned}
$$

with $a$ being the lattice constant. The first term describes the step formation energy $\left(C_{0}\right)$. The second term describes the elastic step interaction energy. At a step on a strained layer, there is a discontinuity in the thickness; hence, the strain energy, which is contained in the

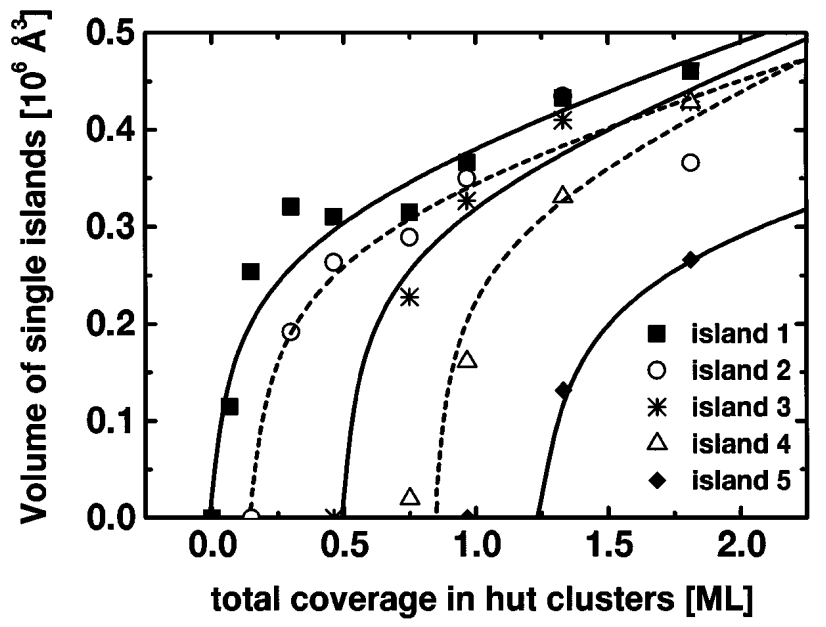

FIG. 2. Evolution of the volume of individual hut clusters. The different symbols correspond to different individual islands as numbered in Fig. 1. The size evolution shows self-limiting behavior: The initially larger growth rate (large slope) just after the nucleation decreases when the islands grow larger. Results of a model calculation of kinetically self-limiting growth including an energy barrier for the nucleation of new material on the facets are shown as solid and dashed lines. thicker part of the strained layer, is larger. The resulting discontinuity in the surface stress creates a "force monopole." According to Ref. [14], the elastic interaction in the strained layer can be described entirely by the interaction of these monopoles. The constant $C_{m}$ is proportional to $(\text { strain } \times \text { step height })^{2}$. The elastic interaction contained in the logarithmic monopole term depends not only on the distance between steps, but also on their orientation; $\sigma_{i}$ is +1 for up steps and -1 for down steps. Thus, two steps with the same orientation have an attractive interaction, while two steps of opposite orientation have a repulsive interaction. The last term in Eq. (1) describes a repulsive short-range dipole interaction between steps. The constant $C_{d}$ was determined by the constraint that the $\{105\}$ facets have a minimum energy.

With a facet angle of only $11.2^{\circ}$, the $\{105\}$ faceted islands are rather flat and can be considered as an array of steps with a step height of $1.4 \AA$ and a terrace width of $l=7 \AA$ as indicated in Figs. 3(a)-3(c). Using a completely filled facet as a reference, the energy for islands with partly filled facets [grey blocks in Figs. 3(b) and 3(c)] will be calculated, and an energy barrier will be found for the nucleation of new facets. The energy of a square shaped hut cluster composed of step-step interaction energies is given in Ref. [5]. Here we treat the more general case of elongated hut clusters. Since perpendicular steps do not interact with each other, we consider first only the interaction between the two small facets [cf. Fig. 3(a)]. As an approximation all steps are considered to have the same length $s$ [5]. Since we consider only energy differences of islands with the same number of steps, the first term in Eq. (1) is a constant and is neglected. Using Eq. (1) and the summation over all step pairs, the energy of the two small faces of a hut cluster can be written as

$$
\begin{array}{r}
E^{\text {small }}(s, k, d)=s \sum_{i=1}^{2 n-1} \sum_{j=i+1}^{2 n}[ \\
C_{m} \sigma_{i} \sigma_{j} \ln \left(\frac{x_{i j}(k, d)}{a}\right) \\
\left.+C_{d}\left(\frac{x_{i j}(k, d)}{a}\right)^{-2}\right],
\end{array}
$$

where $x_{i j}$ is the matrix of step-step distances,

$$
x_{i j}(k, d)=|i-j| l+d g(i, j)+b f(i, j, k),
$$

with $g(i, j)=1$ or 0 if $i$ and $j$ are on different or the same facets, respectively, while $f(i, j, k)=1$ if $[(i \geq$ $k) \wedge(j<k)] \vee[(i<k) \wedge(j \geq k)]$, and $f(i, j, k)=0$ otherwise. The sum in Eq. (2) runs over all possible pairs of steps, and the relevant notation is shown in Fig. 3(a). The matrix $x_{i j}$ [Eq. (3)] gives the distances between step pairs and consists of three parts. The first term considers fully filled facets; the second term is an extension for huts with the elongated length $d$. The last term $b f(i, j, k)$ is an extension for partially filled facets and treats the nucleation of a new facet [grey blocks in Fig. 3(c)]. The 
(a)

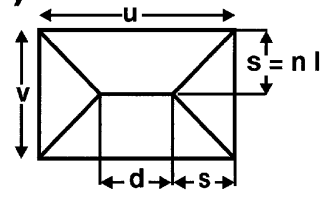

(c)
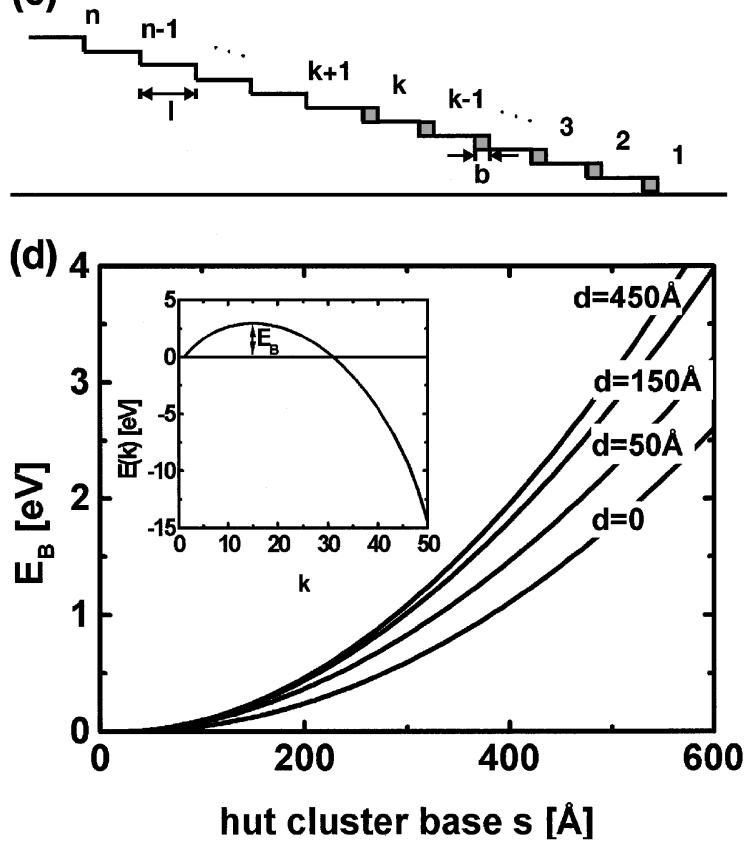

FIG. 3. Plan-view schematic (a) and perspective view (b) of a hut cluster. In (b) the nucleation of a new facet is indicated as a shaded area. (c) Cross section of a facet consisting of individual steps separated by terraces of the width $l$. A partly covered facet (shaded blocks) is completed up to the $k$ th step. (d) The inset shows the island energy as a function of the facet completion $k(d=0)$. An energy barrier $E_{B}$ has to be overcome to nucleate growth on a facet. Panel (d) shows the energy barrier to nucleate a new facet as a function of the cluster base $s$ and the elongation $d$.

growth of a new facet starts at the bottom of the facet. During the growth of a facet, each step ledge is completed before growth continues at the next higher step. An indication of such growth on the facets has been observed by STM [10].

The inset in Fig. 3(d) shows the energy of a square hut cluster during the growth of a new small facet, i.e., as a function of the number of grown blocks $(k)$ on the facet. An energy barrier $E_{B}$, the nucleation energy for the growth of a new facet, has to be overcome, after which the hut cluster energy decreases as the facet approaches completion. The energy barrier $E_{B}$ for elongated islands is plotted as a function of the small facet size $(s)$ for various elongation lengths $(d)$ in Fig. 3(d). The barrier for the nucleation of a new facet increases with the facet size $(s)$ and the elongation $(d)$. This size dependent nucleation barrier for the repeated overgrowth of the $\{105\}$ facets is the reason for the self-limiting growth behavior.
The nucleation barrier for the large facets can be obtained from the barrier of the small facets by a simple scaling property. The energy barrier for the large facets has to be scaled by the mean step length $(s+d)$ relative to the mean step length of the small facets $(s)$. Therefore the nucleation barrier on the large facets $E_{B}^{\text {large }}(s, d)$ can be written as

$$
E_{B}^{\text {large }}(s, d)=(1+d / s) E_{B}^{\text {small }}(s, d=0) .
$$

In the following, we use the above outlined energetic picture of the nucleation on the facets to calculate the growth morphology of hut clusters as a function of time. The growth rate of the small and large facets can be written as

$$
\begin{aligned}
& \dot{d}=\frac{4 b f}{l} s \exp \left(-\frac{E_{B}^{\text {small }}}{k_{B} T}\right), \\
& \dot{s}=\frac{4 b f}{l}(s+d) \exp \left(-\frac{E_{B}^{\text {large }}}{k_{B} T}\right),
\end{aligned}
$$

respectively. The growth rate is proportional to the exponential of the nucleation barrier and the number of nucleation sites $(4 s / l$ on the two small facets). The attempt frequency $f$ is related to the adatom density at the island. By solving Eqs. (5) numerically, the morphological evolution of the hut clusters can be obtained. The attempt frequency $f$ is the only free parameter in the model and is used to adjust the growth rate of islands for different adatom densities at the island. Results of the model calculations for the evolution of the hut cluster volume are shown as solid and dashed lines in Fig. 2 and are in good agreement with the experimental data. The experimentally observed slower growth rate for larger islands is clearly reproduced. This indicates that a kinetic selflimitation is effective during the growth of larger hut clusters. This kinetic self-limitation arises due to an energy barrier for the nucleation of new material on completely filled $\{105\}$ facets.

Experimentally, we observe a linear increase of the island density with coverage, which may influence the growth rate of the islands. If the incomming flux would distribute equally amoung the islands, the average island growth rate should decrease inverse proportional to the island density. While this would lead to a slower growth rate for later nucleating islands (which have many neighbors), we observe, however, an initial fast growth also for the later nucleating islands. In the framework of our kinetic model this can be explained as follows: Only the smaller islands play an active role in competing for the incoming flux. Larger islands are kinetically limited to accept further atoms. This leads to an increased adatom density and to the continued nucleation of new islands.

Next, we will discuss the shape evolution of hut clusters. As an example, Fig. 4 shows the evolution of the length and the width of the hut cluster, marked as island 1 in Fig. 1 as a function of time. Shortly after the 


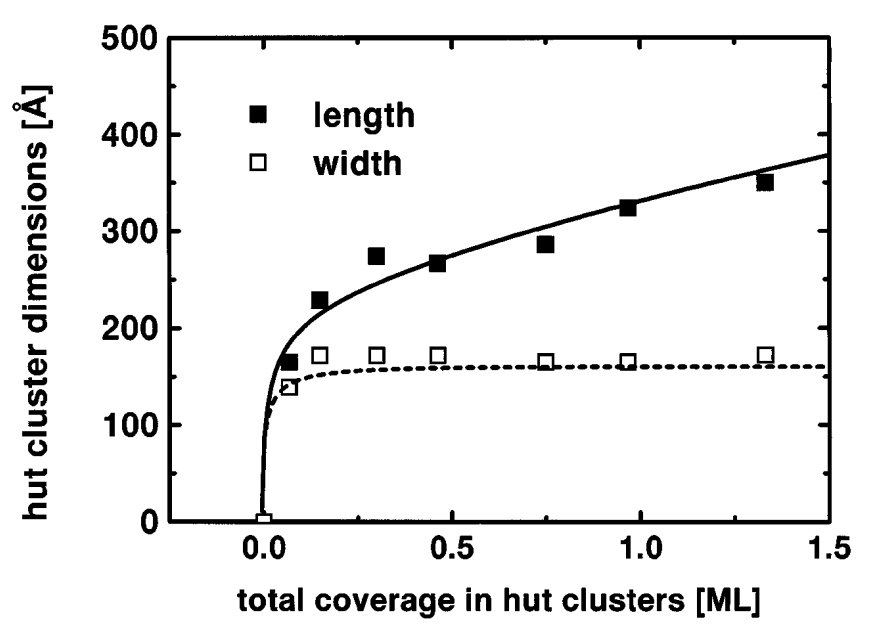

FIG. 4. Length and width of an individual hut cluster as functions of coverage. A shape transition from an initially square shape (length and width area almost equal) to an elongated shape is observed. This transition is predicted by the model for kinetically self-limiting growth, as indicated by the lines.

nucleation the base of the hut clusters is nearly square [as shown for island 1 in Fig. 1(a)], for larger sizes one side (length) becomes longer than the other side (width), leading to a shape transition towards elongated islands (Fig. 4). An energetic explanation for the growth of elongated islands was given in Ref. [15]. However, it is only applicable to islands of constant height. For $\{105\}$ faceted islands the height depends on their width and the energetically stable shape is square $[4,16]$.

The kinetic growth model is used to calculate the shape evolution of hut clusters. Because of the larger size of the nucleus on larger facets, slower growth of the large facets occurs and results in a transition towards elongated shapes. The results of the model calculations are shown as lines in Fig. 4 and correspond well to the experimental data indicated by the symbols. It is also interesting to note that most islands have a quite uniform width (close to $150 \AA$ ), while the length varies strongly (Fig. 1). Also this behavior is reproduced by the growth model (Fig. 4). Growth kinetics as a reason for the elongated growth of the hut clusters is also confirmed by the observation that for lower growth temperatures (below $800 \mathrm{~K}$ ) elongated hut clusters are observed $[7,8]$ and for temperatures higher than $800 \mathrm{~K}$ square shaped hut clusters (equilibrium shape) are observed $[1,11]$.

In conclusion, the evolution of the morphology of $\{105\}$ faceted hut cluster islands has been measured during growth on the nanometer scale and analyzed quantitatively. A model based on a kinetic barrier for nucleation of new facets was applied to model the size evolution and predicted self-limiting growth behavior, which can explain the experimentally observed slower growth for larger islands. The model also predicts the experimentally observed shape transition from initially square shaped islands to elongated islands as they grow larger. These results show how the island morphology is governed by growth kinetics. The kinetically self-limiting growth should favor a narrow island size distribution. However, the observed continuous nucleation of new islands during growth counteracts this trend. We are currently exploring if kinetically self-limited growth of existing islands during coarsening narrows the island size distribution.

Financial support by the DFG Grant No. Vo 711 is gratefully acknowledged.

[1] G. Medeiros-Ribeiro, A. M. Bratkovski, T. I. Kamins, D. A. A. Ohlberg, and R.S. Williams, Science 279, 353 (1998).

[2] J. Tersoff, C. Teichert, and M. G. Lagally, Phys. Rev. Lett. 76, 1675 (1996).

[3] V.A. Shchukin, N. N. Ledentsov, P.S. Kop'ev, and D. Bimberg, Phys. Rev. Lett. 75, 2968 (1995).

[4] V.A. Shchukin, N.N. Ledentsov, M. Grundmann, P.S. Kop'ev, and D. Bimberg, Surf. Sci. 352-354, 117 (1996).

[5] K. M. Chen, D. E. Jesson, S. J. Pennycook, T. Thundat, and R. J. Warmack, Mater. Res. Soc. Symp. Proc. 399, 271 (1996).

[6] D. E. Jesson, G. Chen, K. M. Chen, and S. J. Pennycook, Phys. Rev. Lett. 80, 5156 (1998).

[7] Y.W. Mo, D. E. Savage, B.S. Schwartzentruber, and M. G. Lagally, Phys. Rev. Lett. 65, 1020 (1990).

[8] M. Tomitori, K. Watanabe, M. Kobayashi, and O. Nishikawa, Appl. Surf. Sci. 76/77, 332 (1994).

[9] I. Goldfarb, P. T. Hayden, J.H. G. Owen, and G. A. D. Briggs, Phys. Rev. B 56, 10459 (1997).

[10] I. Goldfarb, J. H. G. Owen, D. R. Bowler, C. M. Goringe, P. T. Hayden, K. Miki, D. G. Pettifor, and G. A. D. Briggs, J. Vac. Sci. Technol. A 16, 1938 (1998).

[11] M. Goryll, L. Vescan, K. Schmidt, S. Mesters, and H. Lüth, Appl. Phys. Lett. 71, 410 (1997).

[12] B. Voigtländer and A. Zinner, Appl. Phys. Lett. 63, 3055 (1993); B. Voigtländer, A. Zinner, and Th. Weber, Rev. Sci. Instrum. 67, 2568 (1996).

[13] Another possible reason for the observed reduced growth rate of larger hut clusters could be the nucleation of a different type of larger islands or islands with dislocations that have a lower chemical potential than the $\{105\}$ faceted islands. As a consequence the adatoms arriving from the vapor would diffuse to the large islands with the lower chemical potential, and the $\{105\}$ faceted islands would grow slower. We can exclude this explanation, since we observe continuous nucleation of new hut clusters up to $2 \mathrm{ML}$ coverage. We would not expect a continued nucleation of new hut clusters if islands outside the area scanned by the STM captured a significant amount of the deposited material.

[14] J. Tersoff, Phys. Rev. Lett. 74, 4962 (1995).

[15] J. Tersoff and R. M. Tromp, Phys. Rev. Lett. 70, 2782 (1993).

[16] J. Tersoff and F. K. LeGoues, Phys. Rev. Lett. 72, 3570 (1994).

[17] World Wide Web: http://www.fz-juelich.de/video/ voigtlaender/ 